Agronomía Costarricense 36(2): 71-79. ISSN:0377-9424 / 2012

www.mag.go.cr/rev_agr/index.html

www.cia.ucr.ac.cr

\title{
MANEJO SOSTENIBLE DE LA BROCA DEL CAFÉ (Hypothenemus hampei) MEDIANTE PODA SISTEMÁTICA DEL CAFETO EN COSTA RICA
}

\author{
Mainor Rojas*
}

Palabras clave: Coffea arabica, broca del café, Hypothenemus hampei, manejo integrado, poda sistemática. Keywords: Coffea arabica, coffee berry borer, Hypothenemus hampei, integrated management, systematic pruning.

\section{RESUMEN}

Se realizaron evaluaciones en 29 cafetales de 9 localidades de Costa Rica, y se condujo un ensayo de campo, con el objetivo de estudiar el comportamiento del ataque de broca en lotes con diferentes sistemas de poda y validar el control diferenciado de la plaga en un sistema de poda por hilera cada 3 años. En sitios con poda por lote cada 3 años, la broca mostró una reducción de $48 \%$ de ataque en las ramas de 1 año respecto a las de 2 años; en lotes con poda por hilera cada 4 años, las ramas de 1 y 2 años mostraron entre 52 y $65 \%$ menos ataque que las ramas de 3 años. Un efecto similar se observó en lotes con poda por hilera cada 3 años, donde las ramas de 1 año presentaron en promedio $56 \%$ menos ataque que las ramas de 2 años. En el ensayo de campo, después de la aplicación diferenciada de insecticida, el porcentaje de frutos con brocas vivas en la hilera de 2 años fue de $0,7 \%$ en ambos tratamientos a los 90 días después de la aplicación; mientras en la hilera de 1 año ese porcentaje fue de $0,7 \%$ donde se aplicó insecticida y $1 \%$ donde no se aplicó, sin mostrar diferencias significativas entre los tratamientos. El control diferenciado de broca, en un sistema de poda por hileras cada 3 años, permitió ahorrar el $30 \%$ del tiempo de la aplicación, $40 \%$ del insecticida y 36,5\% del agua utilizada para la aspersión, al aplicar solo

\begin{abstract}
Sustainable management of coffee berry borer (Hypothenemus hampei) by systematic pruning of coffee plants. Assessments were performed in 29 coffee plantations in 9 locations in Costa Rica and a field trial was conducted, with the purpose of studying the behavior of the coffee berry borer attack in plots with different pruning systems and validating the differentiated pest control in a row-pruning (every 3 years) system. The assessment of the coffee berry borer, in sites where pruning is performed per plot every 3 years, showed a reduction of 48 percent in the attacks to 1-year branches in relation to the 2-year branches; in plots where row pruning is performed every 4 years, attacks were 52 and 65 percent less in 1 and 2-year branches than in 3-year branches. A similar effect was observed in plots with row pruning every 3 years, where 1-year branches had an average of 56 percent less attack than 2-year branches. In the field trial, after the differentiated application of insecticide, the percentage of fruits with coffee berry borers alive in the 2-year row was of 0.7 percent in both treatments at 90 days after application; whereas in the 1-year row this percentage was 0.7 percent where the insecticide was applied and 1 percent where it was not applied, with no significant differences between treatments. The differentiated control of the
\end{abstract}

* Instituto del Café de Costa Rica. Correo electrónico: mrojas@icafe.cr 
el 33\% del cafetal, y sin poner en riesgo el resto del área cultivada. Se concluyó que las podas por lote y por hilera permiten concentrar la población de brocas en áreas reducidas del cafetal, lo que facilita las labores de control.

\section{INTRODUCCIÓN}

El área cultivada con café en Costa Rica al año 2006 se estimó en 98681 ha (ICAFE 2007). La producción nacional de la cosecha 2010-2011 correspondió a 2103287 fanegas (1 fanega=400 litros de café cereza), que generó más de US \$ 383 millones en divisas. Para el año 2010 los ingresos por la exportación de café representaron el $11,77 \%$ del total de divisas generadas por el sector agropecuario nacional (ICAFE 2011).

Entre las plagas insectiles que atacan al café, sobresale la broca del cafeto (Hypothenemus hampei Ferrari), que está catalogada como la de mayor importancia económica, por daños en el cultivo, con capacidad de causar grandes pérdidas a nivel de plantación y grano almacenado, al disminuir el rendimiento, calidad física y organoléptica de la bebida y aumentar los costos de producción y beneficiado del grano.

La presencia de la broca se detectó en Costa Rica en diciembre del 2000. En el 2005 se estimaban 15000 ha con presencia de la plaga (Borbón 2005) y en el 2008 el 92\% del área cultivada (Rojas 2008a). Para el 2011 la dispersión de la broca llegó a cubrir el 97\% del área cafetalera, con un promedio de ataque de $2,76 \%$ a nivel de campo. coffee berry borer, in a pruning system per row performed every 3 years, made it possible to save $30 \%$ in application time, $40 \%$ in insecticide and $36.5 \%$ in water used for aspersion, by making the application to only $33 \%$ of the coffee plantation, and without putting into risk the rest of the area cultivated. It was concluded that pruning per plot, as well as per row, makes it possible to concentrate the coffee berry borer in small areas of the coffee plantation, which contributes to pest control management.

Para el control de la plaga se recomienda el manejo integrado, donde se incluyen las prácticas de control cultural como la regulación de sombra, poda del café, deshija y control de malezas, que dificultan la multiplicación del insecto y facilitan las labores de cosecha y junta. Esas labores se complementan con el control etológico, monitoreo del ataque, control biológico (Beauveria bassiana) y uso del insecticida como última alternativa en casos estrictamente necesarios.

Los ataques de broca en el campo se pueden distribuir de acuerdo con la edad del tejido productivo de la planta y la época de formación de los frutos, lo cual a la vez es influenciado por el sistema de poda utilizado. Se han realizado pocos trabajos para estudiar la relación entre el sistema de poda del cafeto y el ataque de broca.

Los sistemas de poda por lote y poda por hileras se fundamentan en realizar poda baja en lotes completos o hileras completas de cafetos. El ciclo de poda por hileras cada 4 años consiste en dividir cada lote en grupos de 4 hileras para realizar la poda en el orden: 1, 3, 2, 4; se poda el primer año la hilera número 1 , el segundo la número 3 , el tercero la número 2 y el cuarto la número 4. Al quinto año se inicia el ciclo de nuevo en la hilera número 1. Para establecer la poda por lotes la plantación se divide en partes iguales, según la duración del ciclo ( $3,4,5$ o 6 años), a los cuales 
corresponderá la poda baja de acuerdo con un orden preestablecido (Ramírez 1996).

La evaluación del efecto del sistema de poda sobre el comportamiento del ataque aporta información valiosa para adaptar prácticas de manejo de la plaga, como el trampeo y aplicaciones de $B$. bassiana o insecticida, en busca del manejo más eficiente y rentable.

El objetivo de este trabajo fue estudiar el comportamiento del ataque de broca del cafeto en lotes de café con diferentes sistemas de poda y validar el control diferenciado de la plaga en un sistema de poda por hilera cada 3 años.

\section{MATERIALES Y MÉTODOS}

\section{Muestreos de campo}

Los muestreos de ataque de broca se realizaron durante la época lluviosa del 2008 y 2009 en lotes de las variedades Caturra y Catuaí rojo con diferentes sistemas de poda ubicados en Poás, Juan Viñas, Orosi, Cachí, Turrialba, Pérez Zeledón, Barva, Alajuela y Naranjo (Cuadro 1).
El método de muestreo consistió en seleccionar de 10 a 20 plantas al azar bien distribuidas por lote, tomar 100 frutos en forma aleatoria alrededor y a media altura de cada planta seleccionada y calcular el porcentaje de frutos atacados por la plaga, tal como lo indica Rojas (2008b). Para el caso de podas por hilera, el muestreo se realizó para cada edad de ramas en forma independiente.

\section{Ensayo en poda por hilera}

Se condujo durante el 2010 en Cajón de Pérez Zeledón, una de las regiones que ha presentado los mayores ataques de la plaga a nivel nacional. Se estableció a $615 \mathrm{msnm}$, en un suelo Ultisol con 3\% de pendiente, temperatura media anual de $24^{\circ} \mathrm{C}$ y $3000 \mathrm{~mm}$ de precipitación total al año. La variedad utilizada fue Catuaí rojo, con plantas formadas a 2 ejes, distancia de siembra de $1,8 \times 0,9 \mathrm{~m}$, poda por hilera cada 3 años y sombra regulada de Erythrina poeppigiana. Se utilizó una prueba de t pareada con 7 repeticiones (Cuadro 2). La parcela total estuvo constituida por 162 plantas ( 9 hileras de 18 plantas) y la útil por 20 plantas.

Cuadro 1. Evaluación de ataque de broca realizada en lotes con diferentes sistemas de poda. Pérez Zeledón, 2010.

\begin{tabular}{lllc}
\hline \multicolumn{1}{c}{ Evaluación } & \multicolumn{1}{c}{ Método } & Tipo de poda & $\begin{array}{c}\text { Número de } \\
\text { sitios }\end{array}$ \\
\hline $\begin{array}{l}\text { Dispersión a lotes aledaños } \\
\text { a poda total }\end{array}$ & $\begin{array}{l}\text { 10 muestras en hileras 2, 5 } \\
\text { y 10 distantes al lote podado }\end{array}$ & Lote cada 4 años & 3 \\
Ramas 1 y 2 años & 20 muestras por lote & Lote con ciclo cada 3 y 4 años & 6 \\
Ramas 1, 2 y 3 años & 15 muestras por edad & Hilera con ciclo cada 4 años & 8 \\
Ramas 1 y 2 años & 15 muestras por edad & Hilera con ciclo cada 3 años & 12 \\
\hline
\end{tabular}

Cuadro 2. Tratamientos utilizados para evaluar el control diferenciado de broca con Endosulfan 35\% (525 g.i.a.ha ${ }^{-1}$ ) en sistema de poda por hilera con ciclo cada 3 años, Pérez Zeledón, 2010.

\begin{tabular}{lcc}
\hline Tratamiento & Forma de aplicación & Plantas aplicadas.ha $^{-1}$ \\
\hline 1 & Hileras 1 y 2 años & 4115 \\
2 & Hilera 2 años & 2058 \\
\hline
\end{tabular}


Se realizó un muestreo de ataque inicial de broca por parcela y edad de la hilera antes de definir la aplicación del insecticida, con 100 frutos tomados al azar por parcela. El insecticida (Endosulfan 35\%, 525 g.i.a.ha ${ }^{-1}$ ) se aplicó de acuerdo con el tratamiento, con bomba motorizada de espalda. Se realizaron evaluaciones de ataque por parcela y edad de la hilera a los 30, 60 y 90 días después de la aplicación (DDA). A los 90 DDA se disectaron 30 frutos brocados por parcela y edad de la hilera para evaluar la mortalidad. Los datos de porcentaje de ataque y mortalidad fueron transformados $(\sqrt{ } \mathrm{x}+1)$ para realizar el análisis estadístico.

\section{RESULTADOS Y DISCUSIÓN}

\section{Muestreos de campo}

El ataque de broca en los alrededores de lotes donde se realizó poda total el mismo año, fue mayor en las hileras más cercanas al área sin frutos de café, en comparación con las hileras más distantes a 10 y 20 m (Figura 1). De esta manera se obtuvo una reducción promedio entre 58 y $46 \%$ a 10 y $20 \mathrm{~m}$ de distancia, en comparación con la distancia de $4 \mathrm{~m}$ del lote podado.

La evaluación en sitios con poda por lote cada 3 años mostró una reducción promedio de $48 \%$ de ataque en las ramas de 1 año respecto a las de 2 años (Figura 2). En lotes con poda por hilera cada 4 años, las ramas de 1 y 2 años mostraron entre 52 y $65 \%$ menos ataque que las de 3 años (Figura 3). Un efecto similar se observó en lotes con poda por hilera cada 3 años, donde las ramas de un año presentaron en promedio $56 \%$ menos ataque que las de 2 años (Figura 4).

Los resultados de esta investigación muestran que los insectos que quedan en las áreas donde se realizó una poda por lote vuelan hacia las plantas más cercanas, ya que en el área podada no encuentran nuevos frutos. Este comportamiento fue reportado por Castaño et al. (2005), quienes encontraron influencia del vuelo hasta $15 \mathrm{~m}$ del cafetal podado, con mayor ataque en las primeras 4 hileras. Los autores agregaron que la poda por lote rejuvenece la plantación y facilita el control de broca, pero también puede causar una reinfestación muy alta en lotes aledaños.

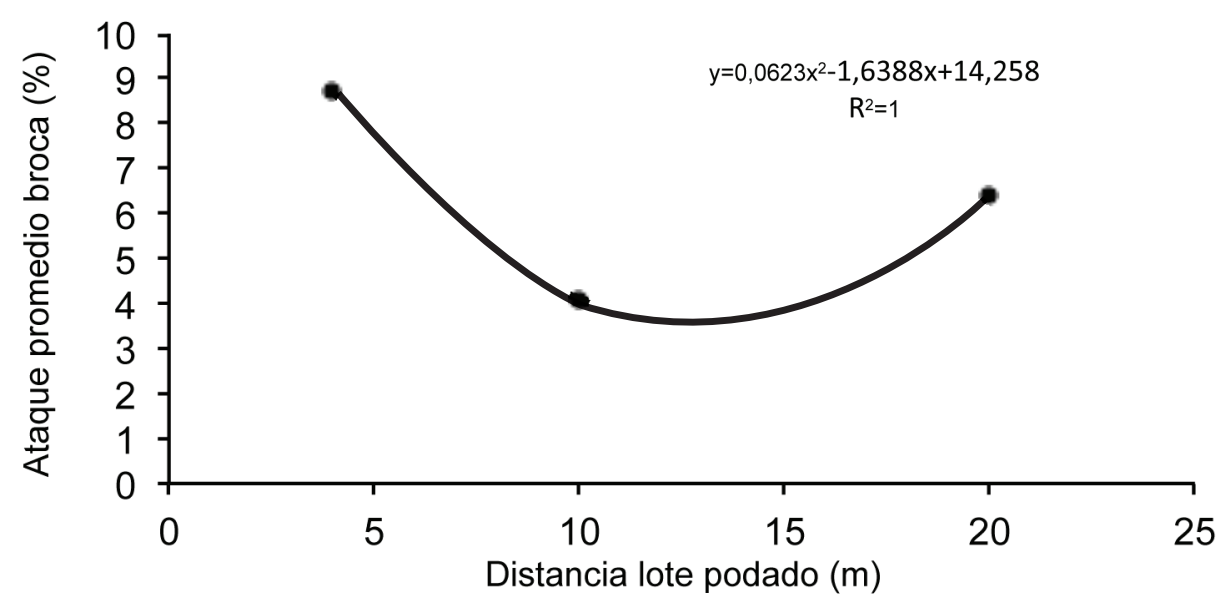

Fig. 1. Porcentaje promedio de ataque de broca de acuerdo con la distancia del lote con poda total. 


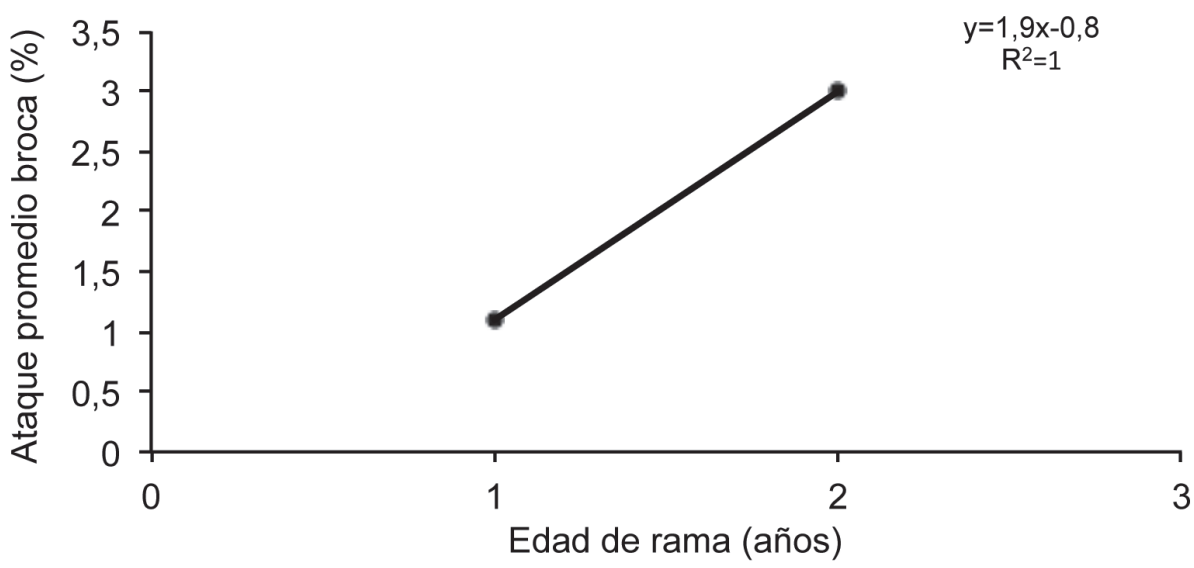

Fig. 2. Porcentaje promedio de ataque de broca de acuerdo con la edad de las ramas en poda por lote cada 3 años.

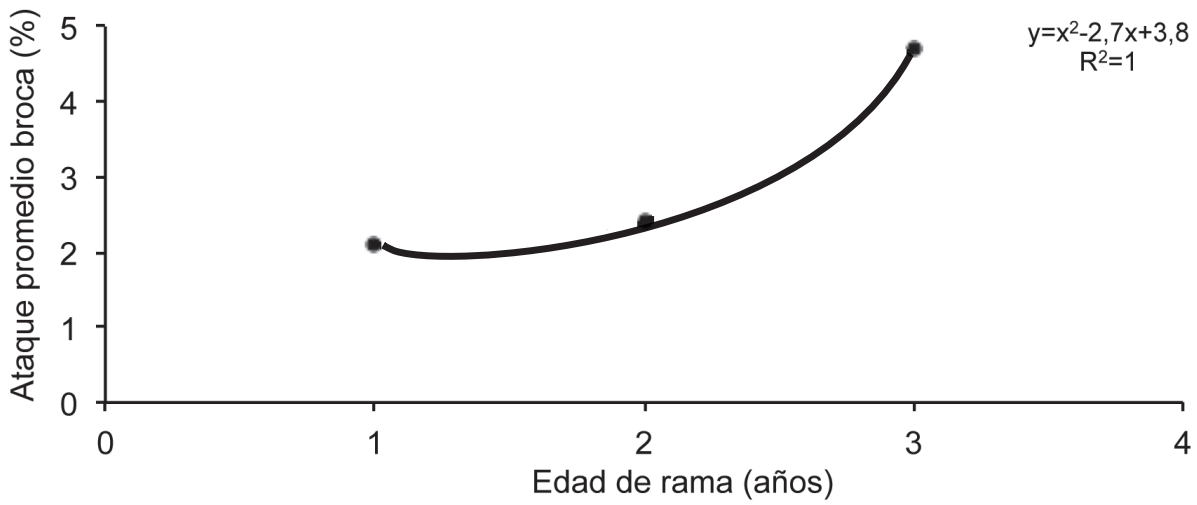

Fig. 3. Porcentaje promedio de ataque de broca de acuerdo con la edad de las ramas en poda por hilera cada 4 años.

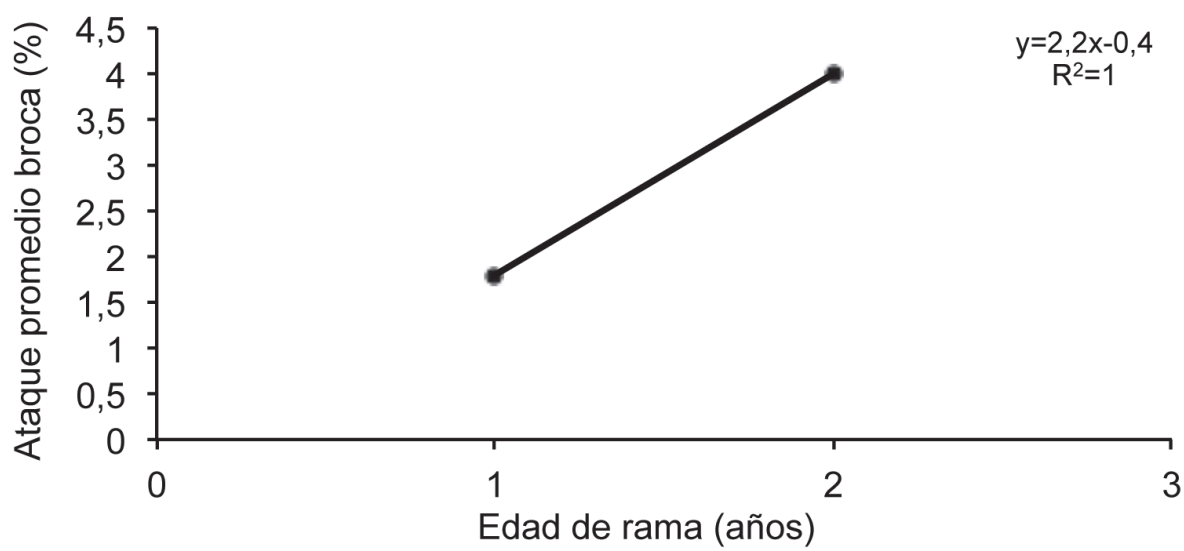

Fig. 4. Porcentaje promedio de ataque de broca de acuerdo con la edad de las ramas en poda por hilera cada 3 años. 
Sobre este problema, es importante conocer el comportamiento del insecto para dirigir el esfuerzo al monitoreo y control de la plaga en esos sitios específicos del cafetal, ya que la poda por lote presenta muchas ventajas para el manejo general de la plantación. Las prácticas de junta y repela aún son fundamentales para reducir la cantidad de brocas que quedan en el cafetal con potencial de atacar la nueva cosecha.

A pesar del ataque inminente que se presenta en las hileras aledañas en una poda por lote, este sistema de poda ofrece la ventaja de concentrar la población de brocas en áreas reducidas, ya que como lo muestran los datos, las ramas de 1 y 2 años presentaron bajos niveles de ataque que no ameritan más que cosechas eficientes. Con este manejo, hasta el tercer año después de la poda se experimentan mayores ataques y en ese momento se aplicarían medidas más estrictas de control, después de verificar su necesidad por medio del monitoreo.

Para la reducción del ataque en las áreas aledañas a un lote podado Bustillo (2007) recomienda remover los frutos brocados de las plantas antes de la poda, usar plantas trampa y cosecharlas
45-60 días después. También recomienda la división de la finca en lotes con diferente edad de ramas, con el fin de poder realizar las labores de control de broca en forma diferencial de acuerdo con el nivel de infestación de la plaga.

El caso de la poda por hilera presentó resultados similares en ciclos de 3 y 4 años, lo que permitió dirigir el control a una tercera o cuarta parte del cafetal en vez de realizar aplicaciones en toda el área lo que contribuye con la reducción de la contaminación del ambiente.

\section{Ensayo en poda por hilera}

En la hilera de 2 años después de la poda el ataque de broca inició alrededor de $8 \%$ y disminuyó hasta llegar por debajo de $4,4 \%$ a los 90 DDA de insecticida en ambos tratamientos, sin mostrar diferencias en ninguna fecha de evaluación (Cuadro 3). En la hilera de 1 año el ataque inició entre 1,6 y 2,7\%, con una tendencia a disminuir en el tratamiento con aplicación de insecticida en esa hilera y a aumentar donde no se aplicó (Cuadro 4).

Cuadro 3. Porcentaje de ataque de broca en la hilera de 2 años de acuerdo con el tratamiento de insecticida en poda por hilera cada 3 años, Pérez Zeledón, 2010.

\begin{tabular}{lcccc}
\hline \multicolumn{1}{c}{ Tratamiento } & \multicolumn{3}{c}{ Porcentaje de ataque de broca } \\
\hline & 0 DDA & 30 DDA & 60 DDA & 90 DDA \\
Aplicación hileras 1 y 2 años & $8,0 \mathrm{~ns}$ & $5,4 \mathrm{~ns}$ & $4,3 \mathrm{~ns}$ & $4,4 \mathrm{~ns}$ \\
Aplicación hilera 2 años & 8,4 & 5,4 & 5,0 & 3,0 \\
\hline
\end{tabular}

Letras diferentes en la misma columna indican que existe diferencia significativa entre tratamientos (Test LSD Fisher Alfa: 0,05).

Cuadro 4. Porcentaje de ataque de broca en la hilera de un año de acuerdo con el tratamiento de insecticida en poda por hilera cada 3 años, Pérez Zeledón, 2010.

\begin{tabular}{ccccc}
\hline \multicolumn{1}{c}{ Tratamiento } & \multicolumn{3}{c}{ Porcentaje de ataque de broca } \\
\hline & 0 DDA & 30 DDA & 60 DDA & 90 DDA \\
Aplicación hileras 1 y 2 años & $1,6 \mathrm{~ns}$ & $2,7 \mathrm{~ns}$ & $1,6 \mathrm{a}$ & $1,4 \mathrm{a}$ \\
Aplicación hilera 2 años & 2,7 & 2,4 & $3,7 \mathrm{~b}$ & $3,1 \mathrm{~b}$ \\
\hline
\end{tabular}

Letras diferentes en la misma columna indican que existe diferencia significativa entre tratamientos (Test LSD Fisher Alfa: 0,05). 
La mortalidad causada por el insecticida a los 90 DDA en la hilera de 2 años fue cercana al $30 \%$ en ambos tratamientos, mientras el porcentaje de frutos abandonados por el insecto se mantuvo entre 46 y 57\%, que llegó a sumar entre 78 y $86 \%$ sin mostrar diferencias significativas $(\mathrm{p}<0,05)$ entre los tratamientos (Cuadro 5). La mortalidad de broca en la hilera de 1 año osciló entre 11 y $27 \%$, el abandono entre 45 y $58 \%$ y la suma de ambas variables entre 70 y $73 \%$, sin mostrar diferencias entre los tratamientos (Cuadro 6).
La variable infestación, que considera solo la presencia de insectos vivos dentro del fruto, no superó el $1 \%$ en las diferentes edades de hilera de cada tratamiento (Cuadro 7) y no presentó diferencias entre ellos $(\mathrm{p}<0,05)$. En el tratamiento donde solo se aplicó la hilera de 2 años se ahorró 0,4 1.ha- ${ }^{-1}$ de insecticida, 115 1.ha ${ }^{-1}$ de agua y $30 \%$ del tiempo experimental para la aplicación (Cuadro 8).

El ataque de broca en la hilera de 2 años al inicio del experimento fue en promedio $74 \%$

Cuadro 5. Mortalidad de brocas y frutos abandonados por el insecto en la hilera de 2 años, 90 DDA de insecticida, Pérez Zeledón, 2010.

\begin{tabular}{cccc}
\hline Tratamiento & \multicolumn{2}{c}{ Porcentaje de mortalidad y abandono } \\
\hline & Mortalidad & Abandono & Suma \\
Aplicación hileras 1 y 2 años & $29,6 \mathrm{~ns}$ & $57,1 \mathrm{~ns}$ & $86,7 \mathrm{~ns}$ \\
Aplicación hilera 2 años & 31,7 & 46,6 & 78,3 \\
\hline
\end{tabular}

Letras diferentes en la misma columna indican que existe diferencia significativa entre tratamientos (Test LSD Fisher Alfa: 0,05).

Cuadro 6. Mortalidad de brocas y frutos abandonados por el insecto en la hilera de un año, 90 DDA de insecticida, Pérez Zeledón, 2010

\begin{tabular}{cccc}
\hline Tratamiento & \multicolumn{2}{c}{ Porcentaje de mortalidad y abandono } \\
\hline & Mortalidad & Abandono & Suma \\
Aplicación hileras 2 y 3 años & $27,6 \mathrm{~ns}$ & $45,8 \mathrm{~ns}$ & $73,4 \mathrm{~ns}$ \\
Aplicación hilera 3 años & 11,2 & 58,6 & 69,9 \\
\hline
\end{tabular}

Letras diferentes en la misma columna indican que existe diferencia significativa entre tratamientos (Test LSD Fisher Alfa: 0,05).

Cuadro 7. Porcentaje de infestación de broca en las hileras de 1 y 2 años 90 DDA, al considerar la mortalidad y el abandono del insecto en cada tratamiento.

\begin{tabular}{ccc}
\hline Tratamiento & Porcentaje de infestación \\
\hline & Hilera 2 años & Hilera 3 años \\
Aplicación hileras 1 y 2 años & $0,7 \mathrm{~ns}$ & $0,7 \mathrm{~ns}$ \\
Aplicación hilera 2 años & 1,0 & 0,7 \\
\hline
\end{tabular}


Cuadro 8. Comparación de cantidad de tiempo, insecticida y agua por hectárea de acuerdo con el tratamiento diferenciado de control químico de broca en sistema de poda por hilera cada 3 años.

\begin{tabular}{cccc}
\hline \multicolumn{1}{c}{ Tratamiento } & $\begin{array}{c}\text { Tiempo aplicación* } \\
\left(\text { h.ha }{ }^{-1}\right)\end{array}$ & $\begin{array}{c}\text { Dosis } \\
\left(\text { L p.c.ha }{ }^{-1}\right)\end{array}$ & Volumen agua (1.ha $\left.{ }^{-1}\right)$ \\
\hline Aplicación hileras 1 y 2 años & 1,43 & 1,0 & 315 \\
Aplicación hilera 2 años & 1,00 & 0,6 & 200 \\
\hline
\end{tabular}

* Tiempo experimental.

mayor que en la hilera de 1 año, aspecto que coincide con los resultados de los muestreos de campo realizados anteriormente a nivel nacional y a su vez reafirma la idea del manejo diferenciado de la plaga de acuerdo con la edad de la rama.

Las evaluaciones realizadas durante 90 días después de la aplicación, hasta poco antes del inicio de la cosecha, mostraron que es posible aplicar insecticida sólo en el 33\% del área del cafetal donde el ataque de broca superaba el 5\%, sin poner en riesgo el resto del área cultivada. El ataque de la plaga en la hilera de 1 año al inicio del ensayo no ameritaba el control químico, ya que apenas superaba el $2 \%$ en promedio y su aplicación aumenta los costos de producción, el riesgo en la salud de los operarios y la contaminación del ambiente.

El ataque evaluado en la hilera de un año sin aplicación de insecticida mostró mayor valor que en la hilera de la misma edad aplicada, pero la infestación al final del ensayo en ambas hileras de los 2 tratamientos no superó el 1\%, nivel muy adecuado para llegar a la cosecha. El porcentaje de frutos abandonados por el insecto fue alto y mostraba que muchos de los frutos en apariencia atacados, realmente no han sufrido daño por parte de la plaga.

Entre otras ventajas, cabe mencionar que la poda por hileras facilita la ejecución de la fertilización y aspersiones en la finca, mejora las condiciones de luminosidad y aireación dentro de la plantación y permite reducir el uso de insumos y mano de obra invertidos en las diferentes labores de mantenimiento de la plantación (Ramírez
1996, Ramírez 2009). Dufour (2008) agrega que el espacio abierto por la poda acelera el desecamiento de los frutos residuales y el desarrollo de las poblaciones de broca.

Además del control adecuado de la plaga, con la aplicación diferenciada en sistemas de poda por hilera cada 3 años, se logró un ahorro importante del tiempo de la aplicación del insecticida y del agua utilizada para la aspersión, además de los otros costos asociados: económicos, ambientales y de salud. Por otro lado, este sistema facilita las labores de recolección y junta del café, recomendadas como parte del manejo integrado.

Castaño et al. (2005) en Colombia reportaron que las brocas que quedaron en los frutos del suelo de un cafetal podado por lote volaron en busca de frutos ubicados en las plantas cercanas, y llegaron a infestar así, las áreas aledañas. Baker (1999) agrega que si una finca en Colombia se maneja con poda por lote, bajo el supuesto de que el lote podado estará rodeado por lotes productivos, será posible que éstos estén sujetos a una invasión masiva de brocas y el manejo de los lotes aledaños se dificulte.

Sobre las observaciones anteriores es necesario considerar que bajo las condiciones de Costa Rica y su cosecha concentrada, en la época que se realiza generalmente la poda ya ha concluido el período de recolección. Por lo tanto, la repela y eliminación de los frutos de la planta al final de la cosecha, son las labores que se deben realizar minuciosamente para evitar infestaciones importantes en los lotes aledaños. 


\section{CONCLUSIONES}

Las podas por lote y por hilera concentran la población de brocas en áreas reducidas del cafetal, lo que facilita así las labores de control.

El control diferenciado de broca en un sistema de poda por hileras cada 3 años permitió ahorrar el 30\% del tiempo de la aplicación, $40 \%$ del insecticida y $36,5 \%$ del agua utilizada para la aspersión, al aplicar solo el 33\% del cafetal y sin poner en riesgo el resto del área cultivada.

\section{LITERATURA CITADA}

BAKER P. 1999. The coffee berry borer in Colombia. Final report of the DFID-Cenicafé-CABI Bioscience IMP for coffee project (CNTR 93/1536A). Chinchiná (Colombia), DFID-CENICAFÉ. 154 p.

BORBÓN O. 2005. Experiencias sobre el manejo de la broca del café en Costa Rica, pp. 58-61. In: J.F. Barrera (ed.). Simposio sobre situación actual y perspectivas de la investigación y manejo de la broca del café en Costa Rica, Cuba, Guatemala y México. Sociedad Mexicana de Entomología y El Colegio de La Frontera Sur.
BUSTILLO A. 2007. El manejo de cafetales y su relación con el control de la broca del café en Colombia. Boletín técnico No. 24. Cenicafé. $40 \mathrm{p}$.

CASTAÑO A., BENAVIDES P., BAKER P. 2005. Dispersión de Hypothenemus hampei en cafetales zoqueados. Cenicafe 56(2):142-150.

DUFOUR B. 2008. Manejo integrado de la broca del café diseñado con tres componentes, pp. 11-15. In: Boletín No. 114, Enero-marzo IICA-PROMECAFE. Guatemala.

ICAFE 2007. Informe sobre la actividad cafetalera de Costa Rica. 52 p.

ICAFE 2011. Informe sobre la actividad cafetalera de Costa Rica. $62 \mathrm{p}$.

RAMÍREZ J. 1996. Poda y manejo de Coffea arabica L. Instituto del Café de Costa Rica, Centro de Investigaciones en Café, Departamento de Investigación y Transferencia de Tecnología en Café. San José, Costa Rica. 60 p.

RAMÍREZ J. 2009. Hacia la caficultura sostenible. Instituto del Café de Costa Rica, Centro de Investigaciones en Café. San José, Costa Rica. 216 p.

ROJAS M. 2008a. Ataque de broca en la cosecha 2007/2008. Revista Informativa (ICAFE) I 2008:10-12.

ROJAS M. 2008b. Muestreo para determinación del ataque de broca: Una buena estrategia para reducir costos de producción. Revista Informativa (ICAFE) II 2008:10-13. 
